\title{
Indicadores de producción de los profesores de Educación Física y Didáctica de la Expresión Corporal en España en la Web of Science
}

Joaquim Reverter Masià

Profesor Titular en el Departamento de Didácticas Específicas de la Universidad de Lleida (España).

Vicenç Hernández González

Profesor Asociado en el Departamento de Didácticas Específicas de la Universidad de Lleida (España)

M. Carme Jové Deltell

Profesora Colaboradora Permanente en el Departamento de Didácticas Específicas de la Universidad de Lleida (España)

Alejandro Legaz Arrese

Profesor Titular en el Departamento de Fisiatría y Enfermería de la Universidad de Zaragoza.

El índice de Hirsch es actualmente uno de los indicadores bibliométricos más utilizados para estimar los éxitos de trabajo de investigadores y predecir el impacto de producción en el futuro. Este trabajo lo utiliza como medida de productividad y calidad científica de las citas totales, número de artículos y Índice $h$ de los profesores españoles de las ciencias del deporte. También se analiza, número de autores por artículo, revistas en que publican, universidad que más artículos publica y profesores que más producen en Web of Science. Los resultados revelan que el Índice $h$ correlaciona ampliamente citas y número de artículos. Las distribuciones por áreas, indican que el área de Educación Física tiene un Índice $h$ medio de 2,65 y el área de Expresión Corporal 1,61. La proporción media de artículos por profesor, oscila entre 4 y 7. La media de autores firmantes oscila entre 4 y 6 . La revista en que más publican los profesores de Expresión Corporal es Revista de Psicología del Deporte y en Educación Física es Journal of Strength and Conditioning Research. Finalmente, se plantean que implicaciones tienen estos 
resultados y el uso que de los mismos se puede dar en la toma de decisiones académicas y de investigación.

Palabras Clave: Profesores; Deporte; Revistas; Web of Science; Bibliometría; Artículos.

\section{Production indicators of teachers of Physical Education and Didactics of Corporal expression in Spain in Web of Science}

Hirsch's h-index has become one of the most widely used bibliometric indicators to assess the success of a researcher working and so predict the impact of future production. In this paper is used as a measure of scientific productivity and quality of total citations, number of items and the index $h$ of Spanish teachers of sports science. Also analyzed the number of authors for paper, in which papers are published journal and more items university professors published and most occur in Web of Science. The results show that the index $h$ broadly correlates with citations and number of papers. The distributions by area, indicate that the Physical Education (PE) area has an Index average of $2.65 \mathrm{~h}$ and 1.61 Didactics of Corporal Expression (DEC) area. The most relevant results are the average proportion of items per teacher in the areas analyzed, between 4 and 7. The average papers authors signing by between 4 and 6. The most published journal Didactics of Corporal Expression teachers is Revista de Psicología del Deporte. In the Physical Education is Journal of Strength and Conditioning Research. Finally, we argue that the implications of these results and the use there of may be given in academic decision making and research.

Keywords: Professors; Sport; Journals; Web of Science; Bibliometric indicators; Papers. 


\section{Produção de professores de Educação Física e Didática de expressão corporal na Espanha, em artigos de revistas com fator de impacto da Web of Science}

O índice de Hirsch é, atualmente, um dos indicadores bibliométricos mais utilizados para avaliar o sucesso do trabalho de pesquisa e prever o impacto da produção futura. Este trabalho foi usado como uma medida da produtividade e da qualidade científica do total de citações, número de itens e índice $H$ de professores de espanhol da ciência do esporte. Também foram analisados o número de autores por artigo, as revistas de publicação e professores universitários com mais publicações incluídas no Web of Science. Os resultados mostram que o índice $h$ correlaciona amplamente compromissos e número de itens. A distribuição por região indica que a área de PE tem uma média de índice de 2,65 h e 1,61 na área de Linguagem. A proporção média de itens por professor está entre 4 e 7 . Os autores possuem média de assinatura entre 4 e 6 . A revista Corpo professores de línguas mais publicados é Jornal de Psicologia do Esporte e do Journal of Strength and Conditioning Research de Educação Física. Finalmente, argumenta-se que as implicações desses resultados e sua utilização podem ser usadas na tomada de decisões acadêmicas e de pesquisa.

Palavras-Chave: Professores; Esportes; Revistas; Web of Science; Bibliometria; Artigos.

Recebido em 14.12. 2012 Aceito em 05.06.2013

\section{Introducción}

La investigación científica tiene gran influencia en el desarrollo económico y social de los países. Sin embargo, los recursos que pueden destinarse a esta actividad son limitados. La demanda de incremento de financiación en investigación ha originado la necesidad de evaluar la actividad científica y su productividad, siendo uno de los criterios más directos las publicaciones científicas (GARCIA-PEREZ, 2000; SALGADO; PÁEZ, 2007; DEVIS et al., 2010; DORTA-GONZÁLEZ; DORTA-GONZÁLEZ, 2010). 
La globalización se ha visto beneficiada por los avances en la comunicación, ha permitido un desarrollo más acelerado en la ciencia. Las revistas de gran prestigio, por ejemplo, las incluidas en la (ISI) Web of Knowledge son consideradas por la comunidad científica de referencia en calidad académica para cada campo de conocimiento (OLIVAS-ÁVILA; MUSI-LECHUGA, 2010; BUELA-CASAL et al., 2010; MUSI-LECHUGA et al., 2005).

Por otra parte, la evaluación de la productividad científica es un ejercicio necesario, ya que de esta manera los investigadores y de manera global las universidades reciben ciertos beneficios a través de las evaluaciones de los organismos institucionales (Agencia Nacional de Evaluación y Prospectiva "ANEP", Comisión Nacional Evaluadora de la Actividad Investigadora"CNEAI" o la Agencia Nacional de Evaluación de la Calidad y Acreditación, "ANECA").

Así pues, de modo general, dos aspectos de la actividad investigadora son tenidos en cuenta por estas agencias y comisiones: la cantidad y la calidad de lo producido. No siempre los indicadores de estos dos aspectos han confluido, dando lugar a numerosos debates al respecto y posiciones encontradas. Los dos criterios fundamentales utilizados en España son, además de la cantidad de artículos publicados (criterio de producción) en revistas indexadas en (ISI) Web of Knowledge, el número de citas que el trabajo de investigación ha recibido y el índice de impacto de la revista en la que se ha publicado el trabajo de investigación (criterio de calidad) (SALGADO; PÁEZ, 2007).

El actual sistema de evaluación y comparación de la producción científica mediante el índice de impacto ha sido criticado desde distintas perspectivas y de manera más patente en estos últimos años, asegurando que publicar en revistas de impacto no implica que dicho trabajo sea de calidad; puesto que en la mayoría de los casos muchos de esos trabajos no reciben ninguna cita o en el mejor de los casos un número muy reducido de ellas, sobre todo durante los primeros años de su publicación (BUELA-CASAL, 2003; COLQUHOUN, 2003; MOED, 2002; SALGADO; PÁEZ, 2007; REVERTER-MASIÀ, 2012).

El factor de impacto de la revista es sólo un indicador de relevancia de la revista en la que se publica un documento, en este sentido una de las preocupaciones más significativas ha sido que sea más relevante la revista que el propio artículo (BROOKFIELD , 2003).

El factor de impacto presenta algunas limitaciones que hay que tener en cuenta, la primera, que solo contempla las citas de los dos o tres años anteriores (respectivamente) y la segunda que no tiene en consideración el impacto de la revista donde se producen las citas de los trabajos (BUELA-CASAL, 2003).

Hay que considerar también las grandes diferencias que existen entre el rango de citas, así como el impacto entre las disciplinas. Las citas tienden a crecer hasta los 3-5 años en ciencias naturales y hasta los 5-7 años en ciencias sociales. Por ejemplo, el total de citas necesarias para estar en el $1 \%$ de los artículos más citados oscilaba entre 285 citas para 
Biología Molécula y Genética y 32 en Educación según el (ISI) Web of Knowledge (1999). En Psicología y Psiquiatría la media de citas necesarias para estar en el $1 \%$ de artículos más citados era de 100 . Finalmente, se da un error de identificación de citas con una media del 7\%, pudiendo llegar al 30\% (AVITAL; COLLOPY, 2001; VAN RAAN, 2005). Pese a estas limitaciones, los indicadores como el total de artículos publicados o el promedio de citas extraídos del (ISI) Web of Knowledge se han asociado a otros indicadores de producción científica, como la evaluación por pares de la reputación científica en varios estudios y han predicho la obtención de premios Nobel (SIMONTON, 2003; SALGADO; PÁEZ, 2007).

En este contexto, a mediados de 2005 apareció un trabajo distinto a los anteriormente mencionados, el bautizado por el profesor de física de la Universidad de California en San Diego como Índice $h$ (HIRSCH 2005). Estudio que generó gran expectación por los autores más destacados en el campo de la evaluación científica (GRUPO SCIMAGO, 2006). J. Hirsch sugería que se podía evaluar la actividad científica de los investigadores y que superaba las limitaciones de otros ampliamente utilizados índices de clasificación de la productividad científica (HIRSCH, 2005).

La idea es sumamente sencilla. Se toman cada uno de los trabajos de un autor y se ordenan de forma descendente en función de las citas que recibe. Cada trabajo ocupa una posición en el ranking según el número de citas recibidas, al que llamamos rango, además de las citas recibidas. De esta manera se construyen dos listas de números, una ascendente (los rangos) y otra descendente (las citas). Cuando los valores de ambas se cruzan, tenemos el Índice $h$. Por lo que el Índice $h$ es una medida de posición, en concreto, aquella en la cual el volumen de citas es menor o igual al número de orden que ocupa el artículo en una distribución descendente de citas (GRUPO SCIMAGO, 2006). Si bien, a pesar de este hecho, es verdad que el Índice $h$ de un científico nunca puede disminuir con el tiempo (SALGADO; PÁEZ, 2007).

Actualmente el Índice $h$ como medida para evaluar el rendimiento investigador parece definitivo, teniendo en cuenta sus debilidades y sus fortalezas. El organismo de financiación de la Comunidad de Madrid (España) ha publicado el uso de Índice $h$ para las evaluaciones institucionales e individuales de calidad de la investigación (RODRÍGUEZ; IMPERIAL, 2007).

En opinión de Braun, Glänzel y Schubert (2005) entre las ventajas del Índice $h$ se encuentran: (a) que es robusto, es decir, es insensible a un exceso accidental de trabajos no citados y también a uno o varios extraordinariamente citados; (b) que combina el efecto de la «cantidad» (número de publicaciones) y la «calidad» (tasa de citas) de un modo equilibrado y específico; y (c) se puede calcular para períodos específicos y no sólo para toda una vida.

Por lo tanto aunque son bastantes los trabajos que han analizado el Índice $h$ con otros parámetros de productividad, ejemplo, el Índice $h$ ha sido aplicado a la literatura de la ciencia de la información por Cronin y Meho (2006), quienes encontraron, como era esperable, una elevada y 
positiva correlación entre $h$ y el número de citas. También ha sido utilizado para comparar la productividad de diferentes equipos de investigación química (VAN RAAN, 2006). En otro contexto, Bornmann y Hans-Dieter (2005) aplican de forma retrospectiva el Índice $h$ a la documentación de más de 400 solicitantes de una beca posdoctoral, estos autores demuestran que el grado de correlación entre el Índice $h$ y la aceptación o rechazo del candidato es significativo.

Que se tenga conocimiento, nunca se a estudiado el Índice $h$ en el área de Educación Física (EF) y de Didáctica de la Expresión Corporal (DEC) en España. Por lo tanto los objetivos del presente estudio serán: presentar el Índice $h$ de los profesores españoles de las áreas de conocimiento de EF y DEC. En segundo lugar, examinar la distribución estadística de dicho índice en esta población y comprobar donde se sitúa la constante de proporcionalidad. En tercer lugar, examinar la relación que hay entre el Índice $h$ y otras conocidas medidas utilizadas en España para evaluar la productividad investigadora, como el número de artículos y índice de citas. Y por último, comparar las dos áreas de conocimiento, en relación a revistas en las que se publica, media de autores por artículo y evolución en ISI Web of Knowledge.

\section{Método}

\subsection{Diseño}

Se trata de un estudio descriptivo, comparativo y correlacional de análisis de documentos. Se tomó en cuenta las consideraciones propuestas por Hirsch (2005). Se tomó en cuenta las consideraciones propuestas por Ramos-Álvarez et al. (2008), las recomendaciones recogidas en los Principios de Berlín para Rankings de Instituciones de Educación Superior (INTERNACIONAL RANKING EXPERT GROUP, 2006) y el estudio de Olivas-Ávila y Musi-Lechuga, (2010).

\subsection{Participantes}

La muestra estuvo formada por 1.469 profesores pertenecientes al área de DEC y EF, de los cuales 412 fueron del área de DEC y 1.057 del área EF.

\subsection{Materiales}

a)Páginas Web de los departamentos del área de DEC y EF;

b)Base de datos (ISI) Web of Knowledge. Esta se encuentra disponible a través de la plataforma (ISI) Web of Knowledge y contiene información sobre investigación multidisciplinaria en revistas para las cuales se calcula su factor de impacto. 


\subsection{Procedimiento}

\subsubsection{Número total de artículos y revistas donde publica}

En primer lugar, se seleccionaron los participantes a partir de la información que aparecía en las páginas web de los departamentos de DEC y EF de toda España. En segundo lugar, los datos obtenidos se realizaron en función de los registros recabados en la (ISI) Web of Knowledge, teniendo en cuanta que en muchas ocasiones hay errores en los nombres de los autores (Ejemplo: Author=(Gonzalez, Luis-Millan) AND Address $=($ Univ Valencia $) /$ Author $=($ Gonzalez, LM o Author $=$ (Casajus, Jose A.) AND Author $=($ Casajus, JA)). Se encontró que un mismo autor y un mismo trabajo citado había sido recogido con nombres diferentes) para una revisión más detallada del proceso es importante referirse al estudio de Osca-Lluch, Civera y Peñaranda (2009), donde se mencionan las posibles variaciones en los nombres y apellidos de los profesores sus instituciones de afiliación. Por otra parte, otros acostumbran a unir sus apellidos por medio de un guión, por lo que también se tomó esto en consideración para la búsqueda.

El proceso de recolección de la información se realizó en la base de datos mencionada, ingresando en el apartado de «General Search», en donde se utilizó el campo de «Author» y «Address», incluso en algunos casos se utilizo el campo «Topic». Siempre se ingresaron uno o los dos apellidos del autor, algunos casos con letra mayúscula, en otros en minúscula, a veces seguidos por un espacio y las iniciales del nombre, también se emplearon truncadores y operadores booleanos para garantizar la obtención del total de artículos por autor considerando todas las combinaciones posibles en las que se pueden ordenar los apellidos, iniciales de los nombres e inicial del primer apellido, siguiendo en todos los casos el nombre suministrado por las webs oficiales de la Universidad.

\subsection{Método de cálculo del Índice $h$}

El método de cálculo del Índice $h$ sugerido por Hirsch (2005) y descrito en más detalle por Imperial y Rodríguez-Navarro (2007) consiste en los siguientes pasos: (1) Entrar en Web of Knowledge y elegir la opción «General Search»; (2) escribir el nombre del autor en la ventana correspondiente; (3) cuando el sistema responda la lista de publicaciones, utilizar la opción de clasificación por orden de citas («Sort by times cited «); y (4) a continuación identificar el último trabajo cuyo número de orden sea superior al del número de citas que tiene asociado. Ese número de orden corresponde al Índice $h$ del autor.

\subsection{Número total de citas y cita máxima}

El número total de citas es el sumatorio de todas las citas de los trabajos de un investigador en la base de datos mencionada. La cita 
máxima corresponde al trabajo que ha sido citado el mayor número de veces de entre los mencionados de un autor.

Las búsquedas se llevaron a cabo durante los meses de noviembre de 2011 hasta diciembre de 2011 por dos investigadores de manera independiente con la finalidad de obtener mayor fiabilidad de los resultados. Finalmente, se debe considerar que puede existir una variación al momento de consultar los resultados en el presente estudio y esto es debido principalmente a que la base de datos (ISI) Web of Knowledge no presenta reportes mensuales con resultados estáticos, por el contrario, se caracteriza por ser dinámica ya que se agrega información de manera constante, el número de registros puede variar de un día a otro.

\section{Resultados}

Se analizaron un total de 1469 profesores, de los cuales 412 corresponden al área de DEC y 1057 del área EF. De los 412 profesores del área de DEC 65 profesores tenían artículos en la base de datos Web of Knowledge $(15,77 \%)$ y de los 1057 profesores del área de EF, 256 profesores tenían artículos en la base de datos Web of Knowledge $(24,21 \%)$.

\subsection{El Índice $h$ y profesores con mayor producción por áreas académicas}

En la Figura 1 se muestran la producción de los profesores en cada área académica, el número de registros de artículos con el que cuentan, el número de citas que tienen, número de revistas en que publican y el Índice $h$. Resaltar que los ocho profesores con el índice $h$ más elevado del área de EF tienen una media de 13,75, mientras que el resto de la muestra obtiene una media de 2,65 de Índice $h$. En cuanto al área de DEC, no sucede lo mismo, ya que el rango de valores del Índice $h$ oscila entre 5 y 1 .

Respecto a los datos correspondientes al total de citas que cada uno de los profesores acumula al sumar las citas que figuran en el $W e b$ of Knowledge puede observarse que el rango de valores en EF oscila entre 1490 y 1, con un valor promedio de 40,02 citas. En el área de DEC que el rango de valores oscila entre 117 y 1 , con un valor promedio de 12,82 citas.

Comparando ambas áreas puede observarse que, globalmente consideradas, los profesores que tienen una $h$ más elevada son los que tienen también un mayor número de citas a su trabajo.

Usando los datos de las dos áreas puede establecerse, por tanto, que el perfil medio de un profesor de EF con publicaciones en Web of Knowledge en España tendrá al menos una $h$ de 2,65 y más de 40,02 citas a su trabajo. Un profesor de DEC con publicaciones en Web of Knowledge en España tendrá al menos una $h$ de 1,61 y más de 12,82 
citas a su trabajo. Con estos datos podemos establecer que la constante de proporcionalidad en el área de EF es de 2,65 y en el área de DEC es 1.61 (Tabla 1).

En el área de $E F$, se confirma que la distribución de los indicadores de citas responde a los criterios de concentración asimétrica. La distribución es asimétrica, ya que sólo un tercio $(35,26 \%)$ de los autores están por encima de la media en cuanto a Índice $h$ se refiere, y un $24,10 \%$ de los autores están por encima de la media en $\mathrm{n}^{0}$ de citas. Cabe destacar, que 10 autores con el Índice $h$ mayor, un 4,46\%, son responsables del $48,56 \%$ de las citas.

En el área de DEC el 43,85 \% de los autores están por encima de la media en cuanto a Índice $h$, lo que supone prácticamente la mitad de la muestra, un $24,56 \%$ de los autores están por encima de la media en no de citas de sus trabajos, y en este caso coincide con el área de EF. Recalcar también, que los diez autores con el Índice $h$ más alto, es decir un $17,54 \%$ son responsables del $63,74 \%$ de las citas. 
Indicadores de producción de los profesores de Educación Física y Didáctica de la Expresión Corporal en España en la Web of Science
Joaquim Reverter Masià, Vicenç Hernández González, M.Carme Jové Deltell, Alejandro Legaz Arrese

Figura 1 - No de artículos, no de revistas, no de citas y Índice $\mathrm{h}$ en la áreas de EF y DEC

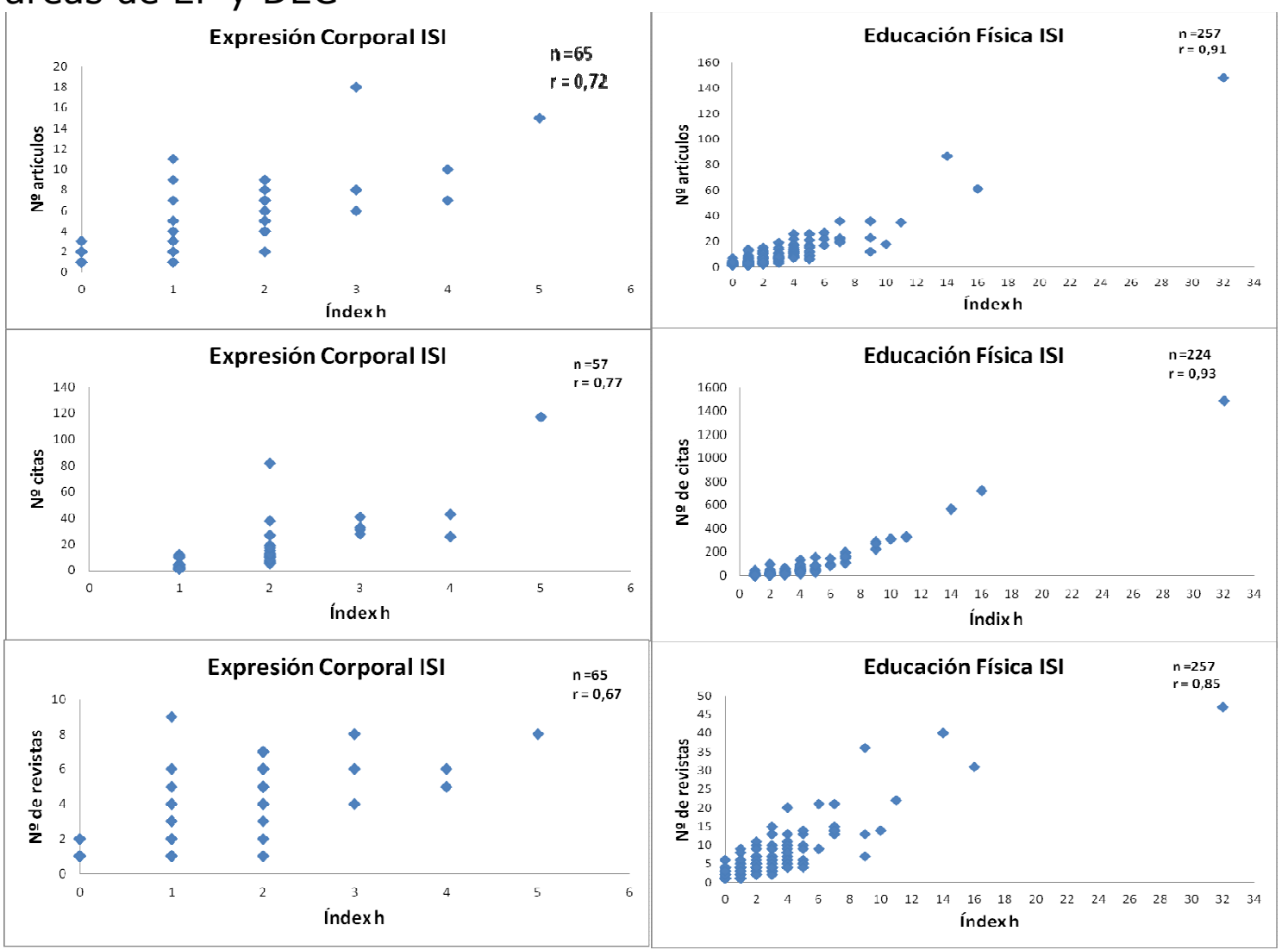

Fuente: Elaboración propia.

En cuanto al número de revistas que se publica, estas varían, como medida estándar podemos afirmar que dos artículos del mismo autor, se publican en la misma revista (Figura 1 ).

El área con mayor producción es la $E F$, donde un 21,19\% del profesorado tiene Índice h. En el área de DEC tienen Índice h el 13,83\% del profesorado (Tabla 1).

Tabla 1 - Índice $h$ y $n^{\circ}$ de artículos en Web of Knowledge de los profesores españoles del área de EF y DEC

\begin{tabular}{cccc}
\hline $\begin{array}{c}\text { Área de } \\
\text { Conocimiento }\end{array}$ & $\begin{array}{c}\text { No Total } \\
\text { Profesores }\end{array}$ & & \\
\hline \multirow{2}{*}{$\begin{array}{c}\text { Didáctica de la } \\
\text { Expresión }\end{array}$} & \multirow{3}{*}{$\begin{array}{c}\text { № Profesores con artículos } \\
\text { Corporal }\end{array}$} & № Profesores con índice & 65 \\
& & Total índice h & 57 \\
& & Media índice h & 93 \\
Educación Física & \multirow{2}{*}{1057} & № Profesores con artículos & 1,63 \\
& & № Profesores con índice & 257 \\
& & Total índice h & 224 \\
& & Media índice h & 594 \\
\hline
\end{tabular}

Fuente: Elaboración propia. 


\subsection{Convergencia entre los indicadores de la productividad y calidad científica}

En la Tabla 2 pueden verse las correlaciones entre los distintos indicadores utilizados en este estudio. De acuerdo con los objetivos, si atendemos a la relación del Índice $h$ con el total de citas de cada investigado, hemos podido observar que en el presente caso la correlación es positiva tanto en el área de DEC como en el área de EF (.77 y .93 respectivamente), y lo mismo ocurre con la correlación del Índice $h$ con el número de artículos por investigador. En ambos casos la magnitud de la correlación es elevada (.72 y .91). Por otra parte también se da una correlación muy elevada en cuanto al número de revistas en que se publica y Índice $h$.

Tabla 2 - Correlaciones entre Índice $h$, el total de citas (NC), no de artículos (NA) y no de revistas (NR) en WoS

\begin{tabular}{|c|c|c|c|c|c|}
\hline \multicolumn{6}{|c|}{ CORRELACIONES } \\
\hline \multicolumn{3}{|c|}{ EXPRESIÓN CORPORAL } & \multicolumn{3}{|c|}{ EDUCACIÓN FÍSICA } \\
\hline $\begin{array}{c}\text { Índice } h x \\
N A\end{array}$ & Índice $h \times N C$ & Índice $h \times N R$ & Índice $h \times N A$ & $\begin{array}{c}\text { Índice } h x \\
N C\end{array}$ & $\begin{array}{c}\text { Índice } h x \\
N R\end{array}$ \\
\hline $0,721674^{*}$ & $0,774025^{*}$ & $0,670903^{*}$ & $0,915824^{*}$ & $0,93993^{*}$ & $0,852785^{\star}$ \\
\hline
\end{tabular}

Nota: ${ }^{*}=\mathrm{p}<.0001 ; \boldsymbol{h}=$ Índice de Hirsch; NC=número total de citas; NA=número total de artículos; $\mathbf{N R}=$ número de revistas

Fuente: Elaboración propia.

\subsection{Revistas con mayor número de registros de artículos por áreas}

Analizando la producción por áreas se encontró que la mayor variedad de revistas registradas en la base de datos Web of Knowledge en las que publican el profesorado se encuentra dentro del área de EF. Las revistas donde más frecuentemente publican los profesores en esta área es el Journal of Strength and Conditioning Research con más de 131 artículos publicados, mientras que la Revista de Psicología del Deporte tiene 101 artículos, por el contrario, los profesores del área de DEC publican más en la Revista de Psicología del Deporte con 28 registros y la Revista Internacional de Medicina y Ciencias de la Actividad Física y del deporte con 27 registros. Cabe destacar, que las 10 primeras revistas en número de registros para cada área de conocimiento, son prácticamente coincidentes.

Tabla 3 - Revistas registradas en la base de Web of Knowledge con mayor número de registros en el área de DEC y en el área de EF

\begin{tabular}{lclcc}
\hline \multicolumn{1}{c}{ Expresión Corporal } & & \multicolumn{2}{c}{ Educación Física } & № \\
\hline \multicolumn{1}{c}{ Revista } & $\begin{array}{c}\text { № } \\
\text { registros }\end{array}$ & Revista & $\begin{array}{c}\text { Nournal of Strength and } \\
\text { registros }\end{array}$ \\
\hline $\begin{array}{l}\text { Revista de Psicología del Deporte } \\
\text { Revista Internacional de Medicina y } \\
\text { Ciencias de la Actividad Física y del }\end{array}$ & 28 & $\begin{array}{l}\text { Conditioning Research } \\
\text { Revista de Psicología del Deporte }\end{array}$ & 131 \\
\hline
\end{tabular}


Indicadores de producción de los profesores de Educación Física y Didáctica de la Expresión Corporal en España en la Web of Science
Joaquim Reverter Masià, Vicenç Hernández González, M.Carme Jové Deltell, Alejandro Legaz Arrese

\begin{tabular}{|c|c|c|c|}
\hline \multicolumn{4}{|l|}{ deporte } \\
\hline Perceptual and Motor Skills & 16 & $\begin{array}{l}\text { Medicine and Science in Sports } \\
\text { and Exercise }\end{array}$ & 99 \\
\hline $\begin{array}{l}\text { Journal of Sports Science and } \\
\text { Medicine }\end{array}$ & 13 & Perceptual and Motor Skills & 78 \\
\hline $\begin{array}{l}\text { International Journal of Sports } \\
\text { Medicine }\end{array}$ & 10 & $\begin{array}{l}\text { Journal of Sports Medicine and } \\
\text { Physical Fitness }\end{array}$ & 62 \\
\hline Psycho-Oncology & 9 & $\begin{array}{l}\text { European Journal of Applied } \\
\text { Physiology }\end{array}$ & 56 \\
\hline $\begin{array}{l}\text { Journal of Strength and Conditioning } \\
\text { Research }\end{array}$ & 8 & $\begin{array}{l}\text { Journal of Sports Science and } \\
\text { Medicine }\end{array}$ & 55 \\
\hline Journal of Human Kinetics & 8 & $\begin{array}{l}\text { International Journal of Sports } \\
\text { Medicine }\end{array}$ & 51 \\
\hline $\begin{array}{l}\text { Medicine and Science in Sports and } \\
\text { Exercise }\end{array}$ & 7 & Journal of Sports Science & 42 \\
\hline $\begin{array}{l}\text { Research Quarterly for Exercise and } \\
\text { Sport }\end{array}$ & 7 & $\begin{array}{l}\text { Revista Internacional de Medicina y } \\
\text { Ciencias de la Actividad Física y } \\
\text { del deporte }\end{array}$ & 37 \\
\hline Revista de Educación & 7 & $\begin{array}{l}\text { Journal of Human Movement } \\
\text { Studies }\end{array}$ & 37 \\
\hline Science and Sports & 7 & European Journal of Sport Science & 29 \\
\hline British Journal of Sports Medicine & 6 & Internacional Journal of Obesity & 25 \\
\hline European Journal of Sport Science & 6 & British Journal of Sports Medicine & 24 \\
\hline $\begin{array}{l}\text { Journal of Sports Medicine and } \\
\text { Physical Fitness }\end{array}$ & 5 & Haemophilia & 22 \\
\hline Journal of Sports Science & 4 & $\begin{array}{l}\text { Internacional Journal of Sport } \\
\text { Psychology }\end{array}$ & 23 \\
\hline $\begin{array}{l}\text { Journal of Science and Medicine in } \\
\text { Sport }\end{array}$ & 4 & Nutrición Hospitalaria & 18 \\
\hline Movimiento & 4 & Journal of Applied Physiology & 17 \\
\hline Nutrición Hospitalaria & 4 & $\begin{array}{l}\text { Archives of Physical Medicine and } \\
\text { Rehabilitation }\end{array}$ & 15 \\
\hline Quest & 4 & Psicothema & 13 \\
\hline
\end{tabular}

Fuente: Elaboración propia.

\subsection{Producción total por áreas académicas}

Para el cálculo de la producción media de registros por área analizada se tomó en cuenta el número total de profesorado que a publicado en Web of Knowledge ISI y también el número total de registros identificados como artículos, también en cada área. Como se observa en la Tabla 4, el área de EF tiene una media de 7,72 artículos, mientras que el área de DEC cuenta con una media de 5,79 artículos. El número de autores por artículo en el área de EF es de 5,47 y en el área de DEC es de 4,70 .

Tabla 4 - Producción en artículos de revistas registradas en la base de datos WoS en el área de DEC y en el área de EF

\begin{tabular}{lcccc}
\hline Área & NA & NP & Media & Media AA \\
\hline Educación Física & 1985 & 257 & 7,72 & 5,47 \\
Didáctica de la Expresión Corporal & 371 & 64 & 5,79 & 4,70 \\
\hline
\end{tabular}

$\mathrm{NA}=$ Número de artículos; $\mathrm{NP}=$ Número de profesores; Media $\mathrm{AA}=$ Media Autores por artículo

Fuente: Elaboración propia. 


\subsection{Producción por universidad}

Los datos recabados en la Tabla 5, muestran que en el área de DEC, la Universidad de Cádiz es la que mayor producción de artículos tiene seguida de la Universidad Jaume I de Castellón y la Universidad de Lleida, estas ocupan el segundo y tercer lugar a nivel de producción. Por otra parte, destacar que en las áreas de DEC las Universidades de Valencia, Murcia y Granada son las que tienen más profesorado que publica en revistas de impacto en Web of Science.

En el área de EF son la Universidad de Zaragoza y la Universidad de Canarias las que ocupan el primer lugar, mientras que las que tienen más profesorado que pública en revistas de impacto en WoS son Granada, Politécnica de Madrid y Valencia.

Tabla 5 - Producción por universidades en artículos de revistas registradas en la base de datos Web of Science en el área de Didáctica de la Expresión corporal y área de Educación Física

Expresión Corporal Educación Física

\begin{tabular}{|c|c|c|c|c|c|c|c|}
\hline Universidad & NA & NP & Media & Universidad & NA & NP & Media \\
\hline Cádiz & 33 & 3 & 11,00 & $\begin{array}{l}\text { Zaragoza } \\
\text { Las Palmas de Gran }\end{array}$ & 190 & 8 & 23,75 \\
\hline Jaume I Castellón & 8 & 1 & 8,00 & Canarias & 198 & 9 & 22,00 \\
\hline Lleida & 7 & 1 & 7,00 & INEFC Barcelona & 91 & 7 & 13,00 \\
\hline Murcia & 40 & 6 & 6,67 & León & 117 & 12 & 9,75 \\
\hline Extremadura & 19 & 3 & 6,33 & $\begin{array}{l}\text { Camilo José Cela } \\
\text { Europea Miguel de }\end{array}$ & 37 & 4 & 9,25 \\
\hline Alicante & 15 & 3 & 5,00 & Cervantes & 54 & 6 & 9,00 \\
\hline UOC & 5 & 1 & 5,00 & Castilla la Mancha & 133 & 15 & 8,87 \\
\hline Almería & 19 & 4 & 4,75 & $\begin{array}{l}\text { Politécnica de Madrid } \\
\text { Miguel Hernández de }\end{array}$ & 187 & 22 & 8,50 \\
\hline Valencia & 26 & 6 & 4,33 & Elche & 92 & 12 & 7,67 \\
\hline Islas Baleares & 12 & 3 & 4,00 & Extremadura & 121 & 16 & 7,56 \\
\hline Oviedo & 8 & 2 & 4,00 & Granada & 174 & 25 & 6,96 \\
\hline Jaén & 8 & 2 & 4,00 & Valencia & 145 & 21 & 6,90 \\
\hline Castilla la Mancha & 19 & 5 & 3,80 & Pablo de Olavide & 91 & 15 & 6,07 \\
\hline Huelva & 11 & 3 & 3,67 & A Coruña & 64 & 11 & 5,82 \\
\hline Complutense de Madrid & 10 & 3 & 3,33 & Ramon Llull & 34 & 6 & 5,67 \\
\hline Córdoba & 6 & 2 & 3,00 & Murcia & 59 & 11 & 5,36 \\
\hline País Vasco & 9 & 3 & 3,00 & Vic & 10 & 2 & 5,00 \\
\hline Granada & 17 & 6 & 2,83 & INEFC Lleida & 14 & 3 & 4,67 \\
\hline Autónoma de Barcelona & 2 & 1 & 2,00 & Alicante & 9 & 2 & 4,50 \\
\hline Salamanca & 2 & 1 & 2,00 & Autónoma de Madrid & 66 & 15 & 4,40 \\
\hline Valladolid & 4 & 2 & 2,00 & Vigo & 33 & 9 & 3,67 \\
\hline Zaragoza & 2 & 1 & 2,00 & País Vasco & 17 & 5 & 3,40 \\
\hline Alcalá & 1 & 1 & 1,00 & Sevilla & 21 & 7 & 3,00 \\
\hline Barcelona & 1 & 1 & 1,00 & UCAM & 17 & 6 & 2,83 \\
\hline La Laguna (Canarias) & 1 & 1 & 1,00 & Católica de Valencia & 18 & 7 & 2,57 \\
\hline
\end{tabular}


$\mathrm{NA}=$ Número de artículos; $\mathrm{Np}=$ Número de profesores que publican

Fuente: Elaboración propia.

Como se observa en la Figura 2, las áreas académicas objeto de estudio ha tenido una evolución muy pareja en cuanto a producción de artículos se refiere, se puede ver claramente como las dos áreas tienen un crecimiento exponencial a partir del 2005.

Figura 2 - Evolución de la producción de artículos en revistas registradas en la base de datos Web of Science en las áreas académicas

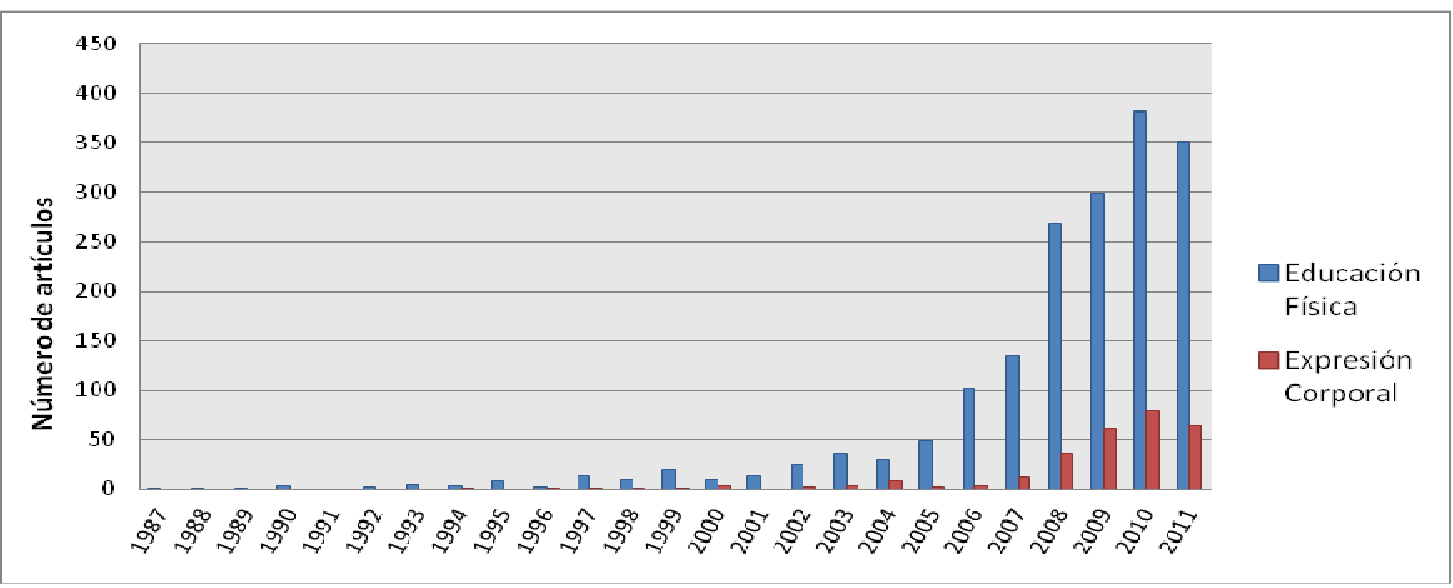

Fuente: Elaboración propia.

\section{Discusión y conclusiones}

Este es el primer estudio, que se tenga conocimiento, que analiza el Índice $h$ y las producciones científicas en las áreas de EF y DEC. De la interpretación de los resultados que aquí se presentan se tiene que tener en cuenta el tipo de muestra utilizada, es decir, sus características y los criterios utilizados para realizar las clasificaciones. Siendo que se trata de la selección de todos los profesores elegidos de acuerdo a la información aparecida en la página WEB de cada facultad, resultaría difícil hacer una comparación con otros estudios, de otras áreas de conocimiento, en que las muestras son mucho menores.

Señala Ball (2005) que los procedimientos de elección de las profesores universitarios y científicos son vistos a menudo como opacos, endogámicos y caprichosos, y que el método de Hirsch podría servir para la toma de decisiones de financiación o de promoción académica de un modo transparente, insesgado y difícil de falsear, porque descansa sobre el conjunto de trabajo realizado a lo largo de muchos años.

En el estudio se pone de manifiesto que existen importantes diferencias en la producción de los profesores del área de EF y el área de DEC en artículos de revistas con factor de impacto en Web of Knowledge, así, los diez autores más productivos en el área de EF, que suponen en total menos del $4,46 \%$ de los profesores del área de conocimiento, consiguen cerca del $25,44 \%$ de la totalidad de los artículos, mientras que 
en el área de DEC, los diez autores más productivos suponen el 17,54\% de la muestra y consiguen el 36,97\% de la producción del área. Es decir, la producción está muy polarizada en un grupo muy reducido de investigadores, los cuales a su vez son los que determinan en buena medida el puesto que ocupa el área de conocimiento en el ranking.

Mediante dicho trabajo, se ha pretendido sondear cual es el potencial del Índice $h$ como un indicador de la calidad de la producción científica de profesores Españoles en Ciencias del Deporte. Los resultados indican que el Índice $h$ es un indicador robusto de la calidad de la investigación. De los resultados se confirma la sugerencia de Hirsch de que las diversas disciplinas podrían tener distribuciones de Índice $h$ diferentes y que las de un dominio científico no pueden ser utilizadas para evaluar otro dominio diferente (SALGADO; PÁEZ, 2007). De manera general, podemos constatar que el Índice $h$ de los profesores de EF y DEC es más bien bajo. Actualmente es necesario un trayecto de largo recorrido para semejarse al tamaño del índice en otros países y otras áreas de conocimiento (GIL SOARES DE ARAUJO; SARDINHA, 2011).

Si hacemos una comparación del Índice $h$ con áreas afines a las analizadas en España, nos encontramos que en un estudio realizado por Salgado y Páez (2007) a los diez catedráticos más productivos de España en Metodología de las Ciencias del Comportamiento tenían un Índice $h$ medio de 4,5; en Personalidad, Evaluación y Tratamientos Psicológicos tenían un Índice $h$ medio de 8,3; Psicología Evolutiva y de la Educación tenían un Índice $h$ medio de 5; Psicobiología tenían un Índice $h$ medio de 14 y en Psicología Social un Índice $h$ medio de 6,5. Por lo tanto teniendo en cuenta que las muestras analizadas son muy diferentes, los resultados de nuestro estudio completarían el estudio realizado por Salgado y Páez (2007) en otras áreas de conocimiento.

Hirsch propone un Índice $h$ de 20 después de veinte años de carrera como un indicador de buen rendimiento científico en el caso de las ciencias físicas. Recordemos que la media de antigüedad de doctorado en ciencias del deporte en España es relativamente reciente. Por tanto, en EF una media de 2,65 para el conjunto de profesores o de 13,75 para los ocho mejores, sólo puede calificarse de aceptable. En cambio, si tenemos en cuenta la muestra total, los resultados son insuficientes, por lo que sugerimos, a los organismos competentes, que se realicen los esfuerzos necesarios para contribuir al avance de la disciplina. En el caso del área de DEC ocurre exactamente igual, aunque hay que tener en cuenta que un porcentaje del profesorado se inicia en las Escuelas Universitarias donde no se les exigía la investigación. Sólo un esfuerzo combinado de reclutamiento de personas motivadas y con normas de alta productividad y de mayor asignación de recursos e incentivos permitiría mejorar el impacto y la productividad científica.

Las revisiones realizadas muestran una tendencia a la estabilización después de un período de desarrollo en la ciencia española en general (FUNDACIÓN CONOCIMIENTO Y DESARROLLO, 2005). La evolución temporal de la producción científica de los profesores españoles tiene un 
crecimiento exponencial año tras año, posiblemente este incremento sea debido a las políticas de las distintas agencias evaluadoras como ANECA, CNEAI y ANEP, no obstante es necesario un mayor esfuerzo.

También hay grandes diferencias en el rango de citas e impacto entre las disciplinas científicas. Las citas tienden a crecer hasta los 3-5 años en ciencias naturales y hasta los 5-7 años en ciencias sociales. Por ejemplo, el total de citas necesarias para estar en el $1 \%$ de los artículos más citados oscilaba entre 285 citas para Biología Molecular y Genética y 32 en Educación según el ISI (1999). En Psicología y Psiquiatría la media de citas necesarias para estar en el $1 \%$ de artículos más citados era de 100. Finalmente, se da un error de identificación de citas con una media del 7\%, pudiendo llegar al 30\% (AVITAL; COLLOPY, 2001; VAN RAAN, 2005). En nuestro estudio la media de citas en el área de EF es de 40,02 y en el área de DEC es de 12,82, por lo tanto los datos estarían en concordancia con los ISI (1999) en ciencias de la educación.

Si tenemos en cuenta la producción por artículos y en número de citas de las dos áreas observamos que los profesores del área de EF producen más y son más citados que los profesores del área de DEC. Atendiendo a los resultados, podemos decir que existe un correlación significativa con el Índice $h$, es decir a más artículos y más citas mayor Índice $h$. Éstos resultados han sido encontrados previamente en diversos estudios en los que se examinaba estas relaciones (SALGADO; PÁEZ, 2007; CRONIN; MEHO, 2006; SIMONTON, 2003).

Las revistas con factor de impacto ISI-Web of Knowledge como criterio de evaluación de las instituciones universitarias es un hecho ampliamente estudiado (CAMPANARIO; CABOS; HIDALGO, 1998; SIERRA et al., 2008), diferentes estudios ponen de manifiesto que la mayor parte de la producción científica española se lleva a cabo por parte de un escaso número de profesores y investigadores (CAMPANARIO; CABOS; HIDALGO, 1998; SIERRA et al., 2008). Nos encontramos con que hay un gran número de profesores titulares y catedráticos de universidad que no tienen artículos indexados en ISI Web of Knowledge (SIERRA et al., 2008). El análisis comparativo entre las dos áreas de conocimiento pone de manifiesto que no hay costumbres de publicar muy diferentes, es decir, que cada investigador publica en la revista que considera más conveniente en función de la temática del artículo, sin haber diferencias entre áreas. En este sentido cabe reseñar que las diez primeras revistas en las dos áreas de conocimiento son prácticamente las mismas. Otro aspecto a tomar en cuenta es el idioma en que publican las revistas, esto puede llegar a condicionar la elección de las mismas a la hora de publicar, y en este sentido, en los últimos años han aparecido varias revistas de origen español que aparecen en un lugar importante en la base de datos Web of Knowledge como son la Revista de Psicología del Deporte y la Revista Internacional de Medicina y Ciencias de la Actividad Física y del Deporte.

Por otra parte, se observa una tendencia cada vez mayor de los profesores españoles a publicar en revistas editadas en inglés esto sin duda influirá en el futuro de las revistas españolas, tal como han 
pronosticado Jiménez-Contreras, Faba y Moya (2001), no obstante es importante mencionar que de las 20 revistas con mayor $\mathrm{n}^{\circ}$ de registros (Tabla 2), en el área de DEC cinco de ellas publican sus artículos en lengua Española, mientras que el área de EF de las 20, cuatro publican artículos en lengua Española.

Greenwood (2007) plantea que el factor de impacto puede ser análogo a un indicador del funcionamiento de una revista. Sin embargo, se debe ser muy cauteloso con la interpretación y el uso de las posiciones de las revistas en el ranking basado en el factor de impacto, puesto que las diferencias en la posición de una revista respecto a otra pueden deberse a diferencias mínimas en los factores de impacto respectivos.

En el campo de las ciencias se reconoce el inglés como el idioma internacional por excelencia (QUINDós, 2009). Ahora bien, esto no puede ser una excusa para nuestros investigadores y permitir que los países de habla inglesa gobiernen en el espacio científico (QUINDÓS, 2009; UGARTE, 2004). Varios autores manifiestan y reclaman, que aquellos trabajos científicos más sobresalientes de nuestros mejores grupos de investigación deberían publicarse, bien en castellano o inglés, pero en nuestras mejores revistas nacionales o supranacionales sin que este hecho comportara una percibida menor calidad de la investigación. Al respecto, diferentes autores creen que deberían hacer una discriminación positiva de todos aquellos artículos escritos en lenguas científicas minoritarias que reciben un número no desdeñable de citaciones bibliográficas (QUINDOS, 2009; UGARTE, 2004; REVETER-MASIA, 2012).

En cuanto a la distribución de revistas por autores, en general hay una baja concentración de artículos en las mismas revistas. Semenzato, Rizzato y Agostini (2004) defienden que hay dos aspectos claves que se deben tener en cuenta a la hora de publicar un artículo, el primer lugar, que sea una revista con factor de impacto Web of Knowledge y en segundo lugar, que tenga el mayor impacto posible por las siguientes razones: estas son las más distribuidas y leídas por la comunidad científica.

En cuanto a la producción por universidad se observan resultados diferentes en las áreas de conocimiento analizadas. Así, por ejemplo, la Universidad de Zaragoza ocupa el primer puesto en EF y la vigésimo segunda en DEC. Por norma general, podemos decir que aquellas universidades que tienen los departamentos de DEC y EF siempre es mucho más productivo uno que otro. Por otro lado, existen grandes diferencias de producción entre las universidades situadas en los primeros puestos de cada área y las situadas en los últimos lugares.

En la evaluación de las universidades, también debería tenerse en cuenta otros aspectos, como las patentes generadas los tipos de investigación desarrollada, el grado de interés de la investigación, la relevancia y la calidad, puesto que muchos investigadores prefieren patentar sus resultados que publicarlos en revistas científicas (BUELACASAL, 2003; SQUIRES, 1992). Sin embargo, y a pesar de la polémica respecto a este tema, los análisis sobre las publicaciones en revistas con 
factor de impacto se utilizan hasta el punto de la aparición de ranking que clasifican las universidades a nivel internacional (SIERRA; BUELA-CASAL; BERMUDEZ, 2008).

Las tres universidades con más producción en artículos con factor de impacto en revistas de Web of Knowledge son Zaragoza, Canarias e INEFC Barcelona en el área de EF y las universidades de Cádiz, Jaume I y Lleida en DEC. En el caso de la Universidad de Cádiz y Zaragoza, era predecible después de ver que tenía varios investigadores entre los más productivos de las áreas de conocimiento. En los países nórdicos, desde hace años, utilizar los artículos con factor de impacto para avaluar a las universidades es una de las premisas para la obtención de recursos económicos (SEGLEN, 1997). Esta misma situación se produce también en países como Canadá y Hungría (SEGLEN, 1997).

En el área de EF la media se sitúa en 7,72 artículos por profesor, mientras que en el área de DEC la media es de 4,36 por profesor. Sin embargo, esta baja producción no se justifica en función del número de revistas con factor de impacto del Journal Citation Reports (JCR), dado que en Sport Science hay cerca de un centenar de revistas especializadas con factor de impacto. Una posible interpretación podría ser que, el área de DEC al tratarse de un área más aplicada que la $E F$, se justificará la tendencia a publicar en revistas más locales (VALENCIANO et al., 2010, REVERTER-MASIA et al., 2012). Estos resultados son similares a los encontrados por Olivas-Ávila y Musi-Lechuga, (2010) en otras áreas de conocimiento, como en Psicología Social $(8,11)$ y Psicología Evolutiva y de la Educación $(6,64)$.

Estos datos, junto con la media de autores por artículo, pueden resultar de gran ayuda a las agencias de evaluación. Los requisitos mínimos, fijados por ANECA (2012), por ejemplo, para obtener la acreditación de titular o catedrático universitario sitúan en 8 y 16 artículos, respectivamente, en $W e b$ of Knowledge. De los resultados obtenidos, también se podría establecer un factor de corrección positiva o negativa en función de los autores firmantes en los artículos.

Este estudio puede resultar de gran utilidad desde distintas perspectivas. En primer lugar, es útil para los profesores, pues tienen un patrón de comparación con el que pueden evaluar, con un criterio real, su producción en artículos con factor de impacto, pero sirve además para establecer y guiar sus metas de promoción a medio y largo plazo. En segundo lugar, resultará especialmente útil a las distintas comisiones y comités que tienen que evaluar la productividad o la selección y promoción de los profesores. En tercer lugar, y posiblemente lo más importante, es que estudios de este tipo permiten a los legisladores de la política científica establecer los niveles de la acreditación para cada nivel de profesorado universitario. 


\section{Referencias}

AGENCIA NACIONAL DE EVALUACIÓN DE LA CALIDAD Y ACREDITACIÓN (ANECA). Principios y orientaciones para la aplicación de los criterios de evaluación, 2012. Disponible en: <http://webs.uvigo.es/esct/pep_nuevo_principiosyorientaciones.pdf $>$. Consultado el: 15 de marzo de 2012.

AVITAL, M.; COLLOPY, F. Assesing research performance: implications for selection and motivation. Sprouts: Working Papers on Information Environments, Systems and Organizations, n. 1, p. 40-61, 2001.

BALL, P. An index for fair ranking of scientists. Nature, n. 436, p. 900, 2005.

BORNMANN, L.; HANS-DIETER, D. Does the h-index for ranking of scientists really work ?. Scientometrics, v. 65, n. 3, p. 391-39, 2005.

BRAUN, T.; GLÄNZEL, W.; SCHUBERT, A. A Hirsch-type index fort journals. The Scientist, n. 19, p. 22, 2005.

BROOKFIELD, J. The system rewards a dishonest approach. Nature, $\mathrm{n}$. 423, p. 480, 2003.

BUELA-CASAL, G. Evaluacion de la calidad de los artículos y de las revistas científicas: propuesta del factor de impacto ponderado y de un índice de calidad. Psicothema, n. 15, p. 23-35, 2003.

BUELA-CASAL, G. et al. Ranking de 2009 en investigación de las universidades públicas españolas. Psicothema, n. 22, p. 171-179, 2010.

CAMPANARIO, J. M.; CABOS, W.; HIDALGO, M. A. El impacto de la producción científica de la Universidad de Alcalá de Henares. Revista Española de Documentación Cientifica, n. 21, p. 402-15, 1998.

COLQUHOUN, D. Challenging the tyranny of impact factors. Nature, $\mathrm{n}$. 423, p. 479, 2003.

CRONIN, B.; MEHO, L. Using the h-index to rank influential information scientists: Brief communication. Journal of the American Society for Information Science and Technology, v. 57, n. 9, p. 1275-1278, 2006.

DEVIS, J. et al. Disciplinas y temas de estudio en las ciencias de la actividad física y el deporte. Revista Internacional de Medicina y Ciencias de la Actividad Física y el Deporte, v. 10, n. 37, p. 150-166, 2010.

DORTA-GONZÁLEZ, P.; DORTA-GONZÁLEZ, M. I. Indicador bibliométrico basado en el índice h. Revista de Documentación Científica, v. 33, n. 2, p. 225-245, 2010.

FUNDACIÓN CONOCIMIENTO Y DESARROLLO. La Universidad en España. Fundación CYD, 2005. Disponible en: < http://www.fundacioncyd.org/publicaciones-cyd/documentos-cyd>.

Consultado el: 22 de abril 2012. 
GARCÍA-PÉREZ, M. A. Assessors' odd listings don't inspire confidence. Nature, n. 406, p. 343, 2000.

GIL SOARES DE ARAUJO, C.; SARDINHA, A. H-Index of the citing articles: a contribution to the evaluation of scientific production of experienced researchers Revista Brasileira de Medicina du Esporte, v. 17, n. 5, p. 358362, 2011.

GREENWOOD, D. C. Reliability of journal impact factor rankings. BMC Medical Research Methodology, n. 7, p. 48, 2007.

GRUPO SCIMAGO. El índice h de Hirsch: aprotaciones a un debate. El professional de la información, v. 15, n. 4, p. 304-306, 2006.

HIRSCH, J. E. An index to quantify an individual's scientific research output. Proceedings of the National Academy of Sciences of the U.S.A., $\mathrm{n}$. 102, p. 16569-16572, 2005.

IMPERIAL, J.; RODRÍGUEZ-NAVARRO, A. La utilidad del índice $\mathrm{h}$ de Hirsch para evaluar la investigación en España. Scientometric, v. 71, n. 2 , p. 271-282, 2007.

INTERNATIONAL RANKING EXPERT GROUP. Berlin principles on ranking of Higher Education Institutions. 2006. Disponible: <http://www.che.de/downloads/Berlin_Principles_IREG_534.pdf>.

Consultado el: 10 de diciembre de 2009.

ISI. Citations reveal concentrated influences: Some fields have it, but what does it means. Science Watch, p. 1-2, 1999.

JIMÉNEZ-CONTRERAS, E.; FABA, C.; MOYA, F. El destino de las revistas científicas nacionales: el caso español a través de una muestra (195090). Revista Española de Documentación Científica, n. 24, p. 147-161, 2001.

MOED, H. F. The impact-factors debate: the ISI's uses and limits. Nature, n. 415, p. 731-732, 2002.

MUSI-LECHUGA, B. et al. Producción de los profesores funcionarios de psicología en España en artículos de revistas con factor de impacto de la Web of Science. Psicothema, n. 15, p. 539-548, 2005.

OLIVAS-ÁVILA, J. A.; MUSI-LECHUGA, B. Análisis de la producción de los profesores funcionarios de Psicología en España en artículos de revistas de la Web of Science. Psicothema, v. 22, n. 4, p. 909-916, 2010.

OSCA-LLUCH, J.; CIVERA M.C.; PEÑARANDA O.M. Consecuencias de los errores en las referencias bibliográficas. El caso de la revista Psicothema. Psicothema, n. 21, p. 300-303, 2009.

QUINDÓS G. Confundiendo al confuso: reflexiones sobre el factor de impacto, el índice h(irsch), el valor Q y otros cofactores que influyen en la felicidad del investigador. Revista Iberoamericana Micología, v. 26, n. 2, p. 97-102, 2009. 
REVERTER-MASIA J. Publicaciones científicas y evaluación de la vida profesional de un científico. Nutrición Hospitalaria, v. 27, n. 4, p. 13681369, 2012.

REVERTER-MASÍA， J.; JOVE-DELTELL, C.; DAZA-SOBRINO， G.; HERNÁNDEZ-GONZÁLEZ, V. Las revistas españolas de Ciencias de la Actividad Física y el Deporte: cómo elegir la revista donde publicar. Educatio siglo $X X I, \mathrm{n}$. 30, p. 217-232, 2012.

RODRÍGUEZ NAVARRO, A.; IMPERIAL RÓDENAS, J. Índice $h$. Guía Para la Evaluación de la Investigación Española en Ciencia y Tecnología Utilizando el Índice h. Madrid: Consejería de Educación de la Comunidad de Madrid. 2007.

SALGADO, J. F.; PÁEZ, D. La productividad científica y el índice $h$ de Hirchs [sic] de la psicología social española: convergencia entre indicadores de productividad y comparación con otras áreas. Psicothema, n. 19 , p. $179-189,2007$.

SEGLEN, P. O. Why the impact factor of journals should not beused for evaluating research. British Medical Journal, n. 314, p. 498-502, 1997.

SEMENZATO, G.; RIZZATO, G.; AGOSTINI, C. Impact factor as measure of scientific quality. American Journal of Respiratory and Critical Care Medicine, n. 169, p. 1070-1, 2004.

SIERRA, J.; BUELA-CASAL, G.; BERMUDEZ, M.P. Análisis transnacional del sistema de evaluación y selección del profesorado universitario. Interciencia, n. 33, p. 251-7, 2008.

SIMONTON, D. K. Scientific creativity as constrained stochastic behavior: The integration of Product, Person and Process Perspective. Psychological Bulletin, n. 129, p. 475-494, 2003.

SQUIRES, B. P. Citation rate: a measure of excellence? Canadian Medical Association Journal, n. 146, p. 341, 1992.

UGARTE, F. La edición de revistas científicas en Latinoamérica. Revista Chilena de Pediatría, n. 75, p. 9-511, 2004.

VALENCIANO, J. et al. La colaboración científica en el campo de las Ciencias de la Actividad Física y el Deporte en España. Revista Española de Documentación Científica, v. 33, n. 1, p. 90-105, 2010.

VAN RAAN, A. F. J. Fatal attraction: conceptual and methodological problemas in the ranking of universities by bibliometric methods. Scientometrics, n. 62, p. 133-143, 2005.

VAN RAAN, A. F. J. Comparison of the $h$-Index with standards bibliometrics indicators and with peers judgments for 147 chemistry research groups. Scientometrics, v. 67, n.3, p. 491-502, 2006. 